the most negative topoisomers migrated close to the major band of positive topoisomers; that is, the two extremities of the arch were close to one another (Fig. 2a). A clear separation of these topoisomers was achieved by introducing chloroquine in the first dimension (Fig. 2b): in these conditions, negative topoisomers were partly relaxed in the first dimension while positive topoisomers were made more supercoiled, so that the whole distribution was shifted to the right of the arch (Fig. $2 b$ ). Furthermore, instead of using chloroquine in the first dimension, the intermediate topoisomers of cellular SSV-1 DNA can be made more supercoiled in vitro by incubation with $S$. acidocaldarius reverse gyrase (Fig. $2 c$ ), an enzyme that creates (but does not remove) positive supercoils ${ }^{7}$. This reaction, which uses ATP, has been described previously with pBR322 as a substrate ${ }^{7}$; these results strongly suggested that the major band of cellular SSV-1 visible at the right end of the arch in Fig. $2 a-c$ comprised highly positive topoisomers. In contrast to cellular DNA, the viral SSV-1 DNA appeared fully positively supercoiled (Fig. $2 d$ ) and the addition of chloroquine to the gel or treatment with reverse gyrase had no effect (not shown).

Other lines of evidence for positive supercoiling include the following. (1) Both DNAs can be relaxed by the action of a eukaryotic type I topoisomerase which relaxes positive and negative supercoils by introducing transient single-strand breaks into the DNA (Fig. $2 f$ ): SSV-1 DNAs are not knotted forms, as knots are only removed by a type II topoisomerase. (2) Partial relaxation of viral SSV-1 DNA produces a set of topoisomers that are positively supercoiled when analysed in a twodimensional gel (Fig. 2e). (3) SSV-1 DNAs are not relaxed by incubation with Escherichia coli topoisomerase I (protein $\omega$ ), which only relaxes negative supercoils (not shown).

Yet another line of evidence derives from the comparison of negatively supercoiled forms of SSV-1 DNA prepared in vitro with natural SSV-1 DNAs. Indeed, it is possible to convert cellular or viral SSV-1 DNAs to negatively supercoiled DNA by incubation with eukaryotic type I topoisomerase in the presence of ethidium bromide; Fig. $2 g, h$ shows two such DNAs with different superhelical densities. In contrast to natural SSV-1 DNAs (Fig. $2 a, d$ ), the migration of these negatively supercoiled DNAs is reduced in a chloroquine gel.

The experiments described here provide strong evidence for the existence of positively supercoiled DNA in vivo. Indeed, we have shown previously that high positive supercoiling can also be achieved in vitro by using the reverse gyrase of Sulfolobus in the presence of polyethylene glycol (PEG) ${ }^{7}$. PEG increases the local concentration of the reactants, providing conditions presumably similar to those existing in vivo. Positive supercoiling of SSV-1 DNA could alternatively be explained by assuming a protein-DNA interaction within the cells that leads to a negatively supertwisted nucleoprotein. After the relaxation of such a complex by a topoisomerase, the removal of the protein in vitro would give rise to a positively supercoiled molecule. This possibility seems unlikely as efficient supercoiling in vitro can be achieved using the reverse gyrase alone in clearly catalytic conditions? ${ }^{7}$.

The observation of the two forms of SSV-1 DNA, one fully supercoiled in the virion, the other found in the bacterium as a wide spectrum of topoisomers (including a small proportion of negative topoisomers), is intriguing. One possible explanation is that within the bacterium, the viral DNA (positively supercoiled) is made negatively supercoiled during transcription and replication, then condensed into a highly positive supercoiled form for encapsidation. The existence of negatively supercoiled and partially relaxed SSV-1 DNA in the bacterium suggests that another topoisomerase, an antagonist of reverse gyrase, is present. An enzyme that can relax positively supercoiled DNA has been identified recently in $S$. acidocaldarius ${ }^{8}$; it is not clear, however, whether this enzyme is a type II topoisomerase analogous to phage $T_{4}$ enzyme, or whether its activity is similar to that of eubacterial gyrase. These different observations raise further questions: is the genome of Sulfolobus itself positively supercoiled (presumably by the activity of reverse gyrase)? Do the variations in the topological state of SSV-1 DNA reflect any topological regulation of the Sulfolobus genome?

The role of reverse gyrase presumably is not restricted to the packaging of viral DNA, as reverse gyrase is abundant in uninfected Sulfolobus strains. Thus, one can hypothesize that the genome (or at least some domains) of Sulfolobus itself is positively supercoiled in vivo and that the level of superhelical density is regulated. Positive supercoiling might be required both to stabilize the DNA in high-temperature $\left(80^{\circ} \mathrm{C}\right)$ growth conditions (to prevent uncontrolled gene expression at this temperature) and to inactivate the genes.

It remains to be determined whether positively supercoiled DNA is restricted to SSV-1 and/or to the genus Sulfolobus, or whether it also exists in other groups of archebacteria, eubacteria and perhaps in some eukaryotes. Recently, a nuclear factor from myeloma cells has been shown to promote positive supercoiling in vitro ${ }^{15}$. As the eukaryotic genome is organized in topologically independent chromatin domains, an attractive hypothesis is that relaxation, or even positive supercoiling, of some of these domains would result in an inactivation of the corresponding genes; this may constitute another mode of gene regulation and expression.

We thank Dr A. Pedrini for protein $\omega$ and Dr J. Trent for critical reading of the manuscript. This work was supported by funds from CNRS (ATP no. 960095).

Received 10 December 1985; accepted 17 March 1986.

1. Wang, J. C. Ann. Rev. Biochem. 54, 665-697 (1985).

2. Drlica, K. Microbiol. Rev. 48, 273-289 (1984)

. Glikin, G. C., Ruberti, I. \& Worcel, A. Cell 37, 33-41 (1984)

4. Pruss, G. J., Manes, S. H. \& Drlica, K. Cell 31, 35-42 (1982)

5. Woese, C. R. \& Fox, G. E. Proc. natn. Acad. Sci. U.S. A. 74, 5088-5090 (1977)

6. Mirambeau, G., Duguet, M. \& Forterre, P. J. molec. Biol. 179, 559-563 (1984).

Forterre, P., Mirambeau, G., Jaxel, C., Nadal, M. \& Duguet, M. EMBO J. 4, 2123-2128 (1985).

8. Nakasu S. \& Kikuchi, A. EMBO J. 4, 2705-2710 (1985)

9. Kikuchi, A. \& Asai, K. Nature 309, 677-681 (1984).

10. Martin, A. et al. EMBO J. 3, 2165-2168 (1984).

11. Zillig, W. et al. System. appl. Microbiol. 7 (in the press)

12. Yeats, S., McWilliam, P. \& Zillig, W. EMBO J. 1, 1035-1038 (1982).

13. Peck, L. J. \& Wang, J. C. Proc, natn. Acad. Sci. U.S.A. 80, 6206-6210 (1983).

14. Pruss, G. J. J. molec. Biol. 185, 51-63 (1985).

15. Lazo, P. Biochem. J. 231, 185-188 (1985).

16. Maniatis, T., Fritsch, E. F. \& Sambrook, J. in Molecular Cloning, 90-91 (Cold Spring Harbor Laboratory, New York, 1982).

17. Duguet, M., Lavenot, C., Harper, F., Mirambeau, G. \& De Recondo, A. M. Nucleic Acids Res. 11, 1050-1075 (1983).

18. Champoux, J. J. \& MacConaughy, B. L. Biochemistry 15, 4638-4642 (1976).

\section{Corrigendum \\ Specific growth response of ras-transformed embryo fibroblasts to tumour promoters}

\section{G. P. Dotto, L. F. Parada \& R. A. Weinberg}

Nature 318, 472-475 (1985)

THIS letter reported that a phorbol ester tumour promoter acted synergistically with an activated ras oncogene to stimulate the outgrowth of transformed foci in early passage rat embryo fibroblast cell cultures. However, the authors neglected to cite an earlier study by Hsiao, W., Gattoni-Celli, S. and Weinstein, I. B. (Science 226, 552-555; 1984) which described a similar phenomenon using the $\mathrm{C} 3 \mathrm{H} 10 \mathrm{~T} 1 / 2$ mouse embryo fibroblast cell line.

\section{Erratum}

\section{The last pluvial climatic episodes in the deserts of southwestern North America}

W. G. Spaulding \& L. J. Graumlich Nature 320, 441-444 (1986)

IN the second paragraph on page 444 , line 4 should read ". . and the Grand Canyon ${ }^{34}, \ldots$... This refers to K. L. Cole's work in ref. 34. In addition, the cover caption to this issue (3 April) moved the site shown (Moctezuma Head 1) to southern California. It is in fact in the Ajo Mountains of southern Arizona. 